proliferation and more importantly less divisions compared with WT animals. FACS analysis of WT and Flt3L $1-$ mice synovium at the acute phase of CIA showed that CD11b+ DC are present and increased in arthritic animals compared with immunised but not arthritic animals. CD103+ DC are only present in WT animals, and increased in animals with a high clinical score.

Conclusions Our data shows that antigen presentation in Flt $3 \mathrm{~L}^{-1}$ mice is impaired. As CD103+ DC are important in presenting and cross-presenting antigens our data reveals an important role for CD103 + DC in both induction and maintenance of CIA. Specifically targeting CD103+ DC could provide a novel antirheumatic strategy.

\section{A9.5 IDENTIFICATION AND VALIDATION OF A PROTEIN COMBINATION INCLUDING S100A9 ABLE TO PREDICT THE RESPONSE TO THE MTX/ETANERCEPT ASSOCIATION IN RHEUMATOID ARTHRITIS PATIENTS}

doi:10.1136/annrheumdis-2013-203223.5

${ }^{1,2} \mathrm{~A}$ Obry, 'T Lequerré, ${ }^{2} \mathrm{~J}$ Hardouin, ${ }^{1,3,4} \mathrm{O}$ Boyer, ${ }^{1,4} \mathrm{O}$ Vittecoq, ${ }^{2 \mathrm{P}}$ Cosette. ${ }^{1}$ INSERM 905, Institute for Biomedical Research, University of Rouen, Rouen; ${ }^{2}$ UMR 6270 CNRS,

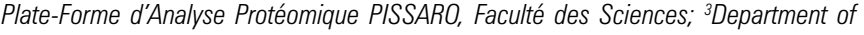
Immunology, Rouen University Hospital; ${ }^{4}$ Department of rheumatology, Rouen University Hospital

Background The number of biologic agents in Rheumatoid Arthritis (RA) is continuously increasing. However, clinicians observe that around 30 to $40 \%$ of treated patients fail to respond to TNF $\alpha$ blocking agents. One way to optimise the drug prescription is to identify predictive markers of drug responsiveness.

Objectives To identify a combination of serum proteins whose expression profile would predict the RA patients responses to the association of methotrexate (MTX) and etanercept (ETA) by mass spectrometry-based quantification methods and ELISA.

Methods A "cohort discovery phase" of 23 patients with active RA was treated by a subcutaneous injection of. The clinical efficacy of these drugs was evaluated with the DAS28 score after 6 months of treatment according to the EULAR response criteria. For proteomic analysis, a serum sample was collected in patients prior to treatment exposure. A "label free" approach on the whole proteome was performed by mass spectrometry on the 25 sera. Accordingly, the proteome of each sample was extracted and in-gel digested. The resulting peptides were analysed by LTO Orbitrap ${ }^{\circledR}$ (ThermoFisher). Differential analysis between responder and non responder samples was performed with LCMS ProGenesis ${ }^{\circledR}$ (Nonlinear Dynamics). To validate these results a relative quantification of selected protein was performed on the second "cohort validation phase" by ELISA. The proteome of peripheral blood mononuclear cells (PBMC) from a second cohort of seven patients with similar characteristics has also been studied by the same label free approach.

Results The label free approach revealed 12 differentially expressed serum proteins according to patient response. This combination of proteins was used to build a Random Forest statistical model to predict the patient's status. This model was validated by a blind test on a panel of seven patients. Moreover, these results have shown the protein S100A9 overexpression in both the serum and the PBMCs from responder's patients and this expression was confirmed by ELISA.

Conclusions The label free approach has identified a combination of predictive markers of response to MTX treatment/ETA. Thus, using sera samples collected in patients prior to treatment exposure, it is possible to predict response to treatment with a small error. These proteins represent interesting candidate biomarkers of response that must be validated in a larger population. Already identified as a diagnostic and prognostic biomarker of RA, the S100A9 protein has been identified as a predictive biomarker of response both in serum and in PBMCs.

\section{A9.6 IDENTIFICATION OF NEW POTENTIAL THERAPEUTIC TARGETS FOR THE TREATMENT OF RHEUMATOID ARTHRITIS: ENTPD1 (CD39) AND 5NTE1 (CD73)}

doi:10.1136/annrheumdis-2013-203223.6

1,2Jonathan D Finn, ${ }^{2}$ Lisa van Baarsen, ${ }^{1,2}$ Jan van Ittersum, ${ }^{1,2}$ Ciska Braam, ${ }^{2}$ Maria C Lebre, 1,2Paul P Tak, 1,2Margriet J Vervoordeldonk. 'Arthrogen BV, Amsterdam, The Netherlands; 'Div. of Clinical Immunology and Rheumatology, Academic Medical Center/University of Amsterdam, Amsterdam, The Netherlands

Background and Objectives Adenosine and ATP are known to have important immunomodulatory properties. Extracellular ATP has multiple roles in inflammation and can act as a damageassociated molecular pattern (DAMP) that can activate the immune system. Conversely, adenosine is primarily anti-inflammatory and can inhibit the production of pro-inflammatory molecules by immune cells. The modulation of ATP and adenosine levels are an essential part of the induction and resolution of an inflammatory response. ENTPD1 (CD39) is a membrane-bound ectonucleoside triphosphate diphosphohydrolase enzyme that converts ATP and ADP to AMP 5NTE1 (CD73) is a 5' ecto-nucleotidase that dephosphorylates AMP to form adenosine. We investigated the role of genes in the adenosine pathway in patients with rheumatoid arthritis (RA) and determined whether expression of CD39 and CD73 would have an effect in an in vitro inflammation model.

Materials and Methods Gene expression analysis using 43k cDNA microarrays (Stanford Functional Genomics Facility) was performed on total RNA extracted from RA synovial tissues obtained by arthroscopy. Adenosine pathway gene expression was compared between high-inflammation versus low-inflammation tissue type synovial biopsies. ATPase levels were measured in synovial fluid (SF) from RA $(n=10)$ or osteoarthritis $(O A)(n=6)$ patients. Adeno-associated viral (AAV) vectors expressing CD39 or CD73 were generated and used to transduce HEK 293 cells or RA fibroblast-like synoviocytes (FLS) and these transduced cells were co-cultured with LPS-activated human monocytes (THP-1) in the presence of ATP. Pro-inflammatory cytokine/chemokine (IL-6, CCL2) production was measured by ELISA.

Results Genes involved in the ATP:adenosine pathway, including CD73, were differentially expressed in high-inflammation synovial tissues, consistent with the hypothesis that there is skewing of the ATP:adenosine balance during inflammation. The half-life of ATP was significantly increased in SF from RA patients compared with $\mathrm{OA}\left(\mathrm{t}_{1 / 2}=8.0\right.$ versus $4.5 \mathrm{~min}$., $\left.\mathrm{p}=<0.05\right)$, indicating that there was a significant decrease in ATPase activity in RA SF HEK 293 cells and RA FLS cell lines transduced with CD39-and/or CD73-expressing AAV5 vectors demonstrated high CD39 and CD73 activity. THP-1 cells stimulated with LPS showed lower levels $(>80 \%$ reduction $p=<0.05)$ of IL- 6 and CCL 2 secretion when co-cultured with CD39 and CD73 expressing HEK293 cells or FLS cells in the presence of ATP.

Conclusions Together, these data suggest that synovial inflammation in RA is characterised by skewing of the ATP:adenosine balance. This could be reversed by overexpression of CD39 or CD73. Thus, these data show that the ATP:adenosine pathway may be a novel therapeutic target for the treatment of RA.

\section{A9.7 LOSS OF PTEN IN MYELOID CELLS CONTROLS INFLAMMATORY BONE DESTRUCTION BY REGULATING THE OSTEOCLASTOGENIC POTENTIAL OF MYELOID CELLS}

doi:10.1136/annrheumdis-2013-203223.7

'Stephan Blüml, ${ }^{2}$ Gernot Schabbauer, ${ }^{2}$ Martin Friedrich, 'Antonia Puchner, 'Victoria Saferding, ${ }^{2} E m i n e$ Sahin, ${ }^{2} T o b i a s$ Lohmeyer, 'Birgit Niederreiter, 'Josef Smolen, ${ }^{1}$ Kurt Redlich. 'Division of Rheumatology, Internal Medicine III, Medical University of Vienna, Austria; ${ }^{2}$ nstitute for Vascular Biology and Thrombosis Research, Center for Biomolecular Medicine and Pharmacology, Medical University Vienna, A-1090 Vienna, Austria; 3 Institute of Immunology, Biomedical Sciences Research Center (BSRC) "Alexander Fleming," Vari, Greece 
Background Local bone destruction in rheumatoid arthritis, psoriasis arthritis or ankylosing spondylitis is a serious health burden and the major cause of disability and severely reduced quality of life in these diseases. This damage to the bony structures is exclusively mediated by a special cell type, the osteoclast (OC). Therefore, it is important to understand factors and pathways regulating the generation of OCs under inflammatory conditions. As PTEN is a lipid phosphatase and one of the main antagonists of the PI3kinase, we analysed the impact of the PI3-Kinase/PTEN axis on OC generation and bone biology in an animal model of inflammatory bone loss.

Methods We induced osteoclastogenesis in wt and PTEN deficient bone marrow cells and measured the generation of OCs, their resorptive capacity and induction of OC differentiation markers in vitro. Moreover, we analysed mice with a monocyte/macrophagespecific deletion of PTEN (myeloid specific $\mathrm{PTEN}^{-/}$) by bone histomorphometry and crossed these mice into hTNFtg animals.

Results We show that myeloid specific PTEN ${ }^{-/}$mice have increased osteoclastogenesis in vitro and in vivo when compared to wild-type animals. However, under non-inflammatory conditions, enhanced osteoclastogenesis did not result in systemic bone loss in vivo. However, when we crossed myeloid specific $\mathrm{PTEN}^{-/}$into hTNFtg mice we found significantly decreased grip strength scores in myeloid specific PTEN $/ / /$ hTNFtg mice compared to wt hTNFtg mice. Joint swelling scores, however, were not different between both groups. In line, myeloid specific PTEN $\% /$ hTNFtg mice displayed enhanced local bone destruction as well as OC formation in the inflamed joints, whereas the extent of synovial inflammation was not different between the groups. Analysis of the synovial membranes of wt and myeloid specific $\mathrm{PTEN}^{-}$animals revealed similar relative compositions of the cellular infiltrate including macrophages, which serve as $O C$ precursors. This suggests that increased capacity for osteoclastogenic differentiation rather than enhanced recruitment of precursor cells is responsible for the enhanced local generation of OCs.

Conclusions Taken together, these data demonstrate that sustained PI3-Kinase activity in myeloid cells specifically elevated the osteoclastogenic potential of these cells, leading to enhanced inflammatory local bone destruction. Therefore, targeting the PI3-Kinase pathway therapeutically may be especially useful for the prevention of structural joint damage.

\section{A9.8 LOW-DOSE IL-2 THERAPY SELECTIVELY EXPANDS REGULATORY T CELLS AND AMELIORATES ESTABLISHED DISEASE IN (NZBXNZW) F1 LUPUS MICE}

doi:10.1136/annrheumdis-2013-203223.8

Jens Y Humrich, Lutz Kloke, Angelika Rose, Caroline von Spee, Anika Klaus, Gabriela Riemekasten. University Hospital Charité, Berlin, Germany

Background Our previous studies in the (NZBxNZW) F1 model provide strong rationales for an IL-2 based immunotherapy of lupus in order to restore regulatory $\mathrm{T}$ cell (Treg) mediated tolerance that is impaired due to an acquired IL-2 deficiency (Humrich et al, 2010). However, because of its pleiotropy, other cells than Treg can be activated by IL-2 in a dose dependent manner, which may induce unwanted side effects or even trigger autoimmunity.

Objectives To determine an optimal regimen for an IL-2 based immunotherapy that is capable to induce a sufficient expansion of CD4+Foxp3+Treg in vivo while only marginally affecting other cells, and that most efficiently influences active disease in the (NZBxNZW) F1 model for lupus.

Methods Recombinant mouse IL-2 at various single doses was injected subcutaneously either into young or diseased (NZBxNZW) F1 mice every day for the duration of five days as induction therapy. After the induction phase, IL-2 injections were continued every 4 days until the end of the experiment. Control animals received an equal amount of PBS (carrier). Cells from lymphoid organs and peripheral blood were analysed by flow cytometry at different time points throughout the study. In addition survival and clinical parameters (weight, proteinuria, leukozyturia, autoantibodies) were analysed during IL-2 therapy of diseased mice for regimens with the single dosages of $5 \mathrm{ng} / \mathrm{g}$ and $25 \mathrm{ng} / \mathrm{g}$ body weight.

Results We found that the low-dose IL-2 regimen with a single dose of $5 \mathrm{ng} / \mathrm{g}$ body weight sufficiently promoted the expansion of CD4+Foxp3+Treg, while not or only marginally affecting CD4+ conventional $\mathrm{T}$ cells (Tcon) and other potentially harmful cells. Although higher doses of IL-2 resulted in a more pronounced proliferation and expansion of Treg, this was accompanied by a considerable increase in CD4+ memory/effector Tcon and NK/NKT cells. Clinically, regimens with both $5 \mathrm{ng} / \mathrm{g}$ and $25 \mathrm{ng} / \mathrm{g}$ were almost equally sufficient to influence nephritis and to decrease mortality in mice with established disease.

Conclusions These studies show that a low-dose IL-2 regimen selectively targets Treg and is clinically effective and also safe in murine lupus providing essential rationales for the clinical introduction of an IL-2 based immunotherapy in SLE.

\section{References}

1. Humrich, JY et al, (2010). Homeostatic imbalance of regulatory and effector T cells due to IL-2 deprivation amplifies murine lupus. Proc Natl Acad Sci USA 107, 204-9.

\section{A9.9 TREATMENT WITH BGP-15, A NOVEL INSULIN SENSITISER ATTENUATES COLLAGEN-INDUCED ARTHRITIS IN DBA/1 MICE}

doi:10.1136/annrheumdis-2013-203223.9

P Mandl, S Hayer, S Blüml, V Saferding, D Sykoutri, J Smolen, K Redlich. Division of Rheumatology, Medical University of Vienna, Vienna, Austria

Background and Objectives BGP-15, a small synthetic hydroxylamine derivative is a member of a new class of insulin-sensitising medications also known as chaperone-inducers. Beside its beneficial effects on glycemic control and insulin sensitivity in patients with Type 2 diabetes, BGP-15 is known to induce heat shock protein Hsp72 and heat shock transcription factor HSF1, which in turn are involved in joint inflammation. Moreover, BGP-15 also inhibits poly-ADP-ribose polymerase (PARP) and the phosphorylation of c-JUN N-terminal kinase via Hsp72 overexpression. Therefore it might also play a role in the regulation of inflammatory joint disease. Our objective was to evaluate the in vivo effects of BGP-15 on collagen-induced arthritis (CIA) in DBA/1 mice.

Materials and Methods Arthritis was induced by intradermal injection of bovine type II collagen (bCII) and incomplete Freund's adjuvant (CFA) in male DBA/1 mice. BGP-15 was administered either one week prior to the first immunisation (prophylactic experiment, $\mathrm{n}: 14$ in both groups) or upon the appearance of symptoms (therapeutic experiment, $\mathrm{n}: 12$ in both groups) in drinking water. Arthritis incidence and severity was assessed for 28 days following the second immunisation (boost) with bCII and CFA on day 21. Histological evaluation was carried out on hind paws using Osteomeasure ${ }^{\circledR}$ software. Anticollagen antibodies were measured by enzyme-linked immunosorbent assay. The cellular composition of the draining lymph nodes was measured by flow cytometry.

Results BGP- 15 significantly reduced the incidence of CIA by $28 \%$ and also reduced both paw swelling $(p \leq 0.01)$ and grip strength $(p \leq 0.05)$ in the prophylactic experiment. In the therapeutic experiment BGP-15 significantly attenuated both paw swelling $(p \leq 0.01)$ and grip strength $(p \leq 0.05)$. Histological evaluation of the hind paws demonstrated reduced area of inflammation $(p \leq 0.05)$, area of erosion $(p \leq 0.01)$ and number of osteoclasts $(p \leq 0.05)$ in the 